This item was submitted to Loughborough's Research Repository by the author.

Items in Figshare are protected by copyright, with all rights reserved, unless otherwise indicated.

\title{
Quantum metamaterials: electromagnetic waves in a Josephson qubit line
}

PLEASE CITE THE PUBLISHED VERSION

http://dx.doi.org/10.1103/PhysRevB.77.144507

\section{PUBLISHER}

(c) The American Physical Society

VERSION

VoR (Version of Record)

LICENCE

CC BY-NC-ND 4.0

\section{REPOSITORY RECORD}

Rakhmanov, Alexander L., Alexandre M. Zagoskin, Sergey Savel'ev, and Franco Nori. 2019. "Quantum Metamaterials: Electromagnetic Waves in a Josephson Qubit Line". figshare.

https://hdl.handle.net/2134/12797. 


\title{
ङु \\ Quantum metamaterials: Electromagnetic waves in a Josephson qubit line
}

\author{
A. L. Rakhmanov, ${ }^{1,2}$ A. M. Zagoskin, ${ }^{1,3,4}$ Sergey Savel'ev, ${ }^{1,3}$ and Franco Nori ${ }^{1,5}$ \\ ${ }^{1}$ Frontier Research System, The Institute of Physical and Chemical Research (RIKEN), Wako-shi, Saitama 351-0198, Japan \\ ${ }^{2}$ Institute for Theoretical and Applied Electrodynamics, Russian Academy of Sciences, 125412 Moscow, Russia \\ ${ }^{3}$ Department of Physics, Loughborough University, Loughborough LE11 3TU, United Kingdom \\ ${ }^{4}$ Department of Physics and Astronomy, The University of British Columbia, Vancouver, British Columbia, Canada V6T $1 Z 1$ \\ ${ }^{5}$ Department of Physics, Center for Theoretical Physics, Applied Physics Program, Center for the Study of Complex Systems, \\ The University of Michigan, Ann Arbor, Michigan 48109-1040, USA
}

(Received 21 October 2007; revised manuscript received 26 January 2008; published 4 April 2008)

\begin{abstract}
We consider the propagation of a classical electromagnetic wave through a transmission line, formed by identical superconducting charge qubits inside a superconducting resonator. Since the qubits can be in a coherent superposition of quantum states, we show that such a system demonstrates interesting effects, such as a "breathing" photonic crystal with an oscillating band gap and a "quantum Archimedean screw" that transports, at an arbitrary controlled velocity, Josephson plasma waves through a transmission line. The key ingredient of these effects is that the optical properties of the Josephson transmission line are controlled by the quantum coherent state of the qubits.
\end{abstract}

DOI: 10.1103/PhysRevB.77.144507

PACS number(s): 74.78.Fk, 74.50.+r, 42.50.-p

\section{INTRODUCTION}

The development of superconducting electronics now allows observations of quantum behavior, such as the coherent superposition of different macroscopic states in mesoscopic and macroscopic devices. ${ }^{1,2}$ While efforts in this field are now mainly directed at the development of superconducting quantum bits as elements of eventual quantum computers, there are other interesting possibilities opened by the existence of such large controllable quantum coherent circuit elements. In particular, analogies with cavity QED have led to interesting theoretical and experimental results. ${ }^{3-8}$ There, qubits play the role of artificial atoms, and high-quality superconducting resonance circuits mimic optical cavities. Differences from cavity QED include the fact that instead of having a stream of identical atoms moving through the cavity, the state of a single qubit that is permanently coupled to the resonator could be periodically changed. In Ref. 9, the results of an earlier experiment ${ }^{10}$ were considered from the point of view of lasing in such a system.

Another recent surge of interest in the electrodynamics of Josephson junction arrays is related to terahertz electromagnetic waves propagating in such systems. Terahertz waves are important for applications but are hardly controllable for both optical and electronic devices. Thus, Josephson structures might be of importance for miniature terahertz generators, filters, detectors, and waveguides. ${ }^{11}$

In this paper, instead of considering a single or a few qubits, we investigate the behavior of an infinite chain of identical qubits inside a resonator from the point of view of terahertz or subterahertz electromagnetic wave propagation in such a quantum medium. We show that by placing the qubits in a quantum superposition state, some interesting possibilities can be realized, including "breathing" photonic crystals and an "Archimedean screw" transport of classical electromagnetic modes.

For lack of a better term, we call such qubit structures, which are considered from the point of view of macroscopic propagation of electromagnetic field, quantum metamaterials. This is because (classical) metamaterials allow additional ways to control the propagation of electromagnetic fields, which are not available to standard materials. (Alternative approaches to superconducting metamaterials were investigated in Refs. 12-18.) Similarly, our proposed quantum metamaterials allow additional ways of controlling the propagation of electromagnetic waves, which are not possible with normal classical structures. Indeed, the coherent quantum dynamics of qubits determines the terahertz "optical" properties in the system.

\section{MODEL}

As a model, we choose a set of identical charge qubits placed at equal intervals $l$ between two bulk superconductors separated by a distance $D$ (Fig. 1). Each qubit is a small superconducting island connected to each superconducting bank by a Josephson junction. The superconducting phase on the $n$th island is $\varphi_{n}$. When treated quantum mechanically, such an island indeed constitutes a qubit if its total capacitance is small enough. ${ }^{1,2}$ The magnetic field $\mathbf{H}$ is applied

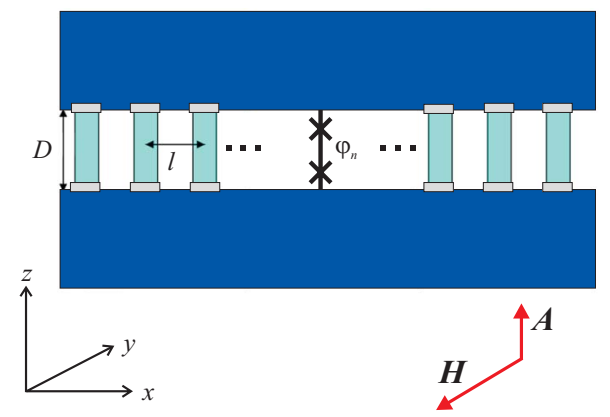

FIG. 1. (Color online) Geometry of the system. Identical charge qubits are placed at equal intervals $l$ between bulk superconductors separated by a distance $D$. 
normal to the structure (in the $y$ direction), and the vector potential A only has a $z$ component. We denote the vector potential between the $n$th and $(n+1)$ th qubits by $A_{z n}$.

The structure in Fig. 1 is a one-dimensional (1D) waveguide with the energy per unit,

$$
\begin{aligned}
\mathcal{E}= & \frac{E_{J}}{2 \omega_{J}^{2}}\left[\left(\frac{2 \pi D \dot{A}_{z n}}{\Phi_{0}}+\dot{\varphi}_{n}\right)^{2}+\left(\frac{2 \pi D \dot{A}_{z n}}{\Phi_{0}}-\dot{\varphi}_{n}\right)^{2}\right] \\
& -E_{J}\left\{\cos \left[\varphi_{n}+\frac{2 \pi D A_{z n}}{\Phi_{0}}\right]+\cos \left[\varphi_{n}-\frac{2 \pi D A_{z n}}{\Phi_{0}}\right]\right\} \\
& +\frac{D l}{8 \pi}\left(\frac{A_{z n+1}-A_{z n}}{l}\right)^{2}+2 I_{n} \varphi_{n} .
\end{aligned}
$$

Here, the dot denotes $\partial / \partial t$. $I_{n}$ is current through the $n$th junction. $\quad E_{J}=\Phi_{0} I_{c} / 2 \pi c, \quad \omega_{J}^{2}=2 e I_{c} / \hbar C, \quad I_{c}, C, \quad$ and $\Phi_{0}$ $=h c / 2 e$ are the Josephson energy, Josephson frequency, critical current, junction capacity, and flux quantum, respectively. We take into account the fact that in the presence of the vector potential, the superconducting phase differences across the junctions of the $n$th qubit $\pm \varphi_{n}$ acquire a gauge term, $\alpha_{n}=2 \pi D A_{z n} / \Phi_{0}$. Introducing the dimensionless units $E=\mathcal{E} / E_{J}$ and $t \rightarrow \omega_{J} t$, we rewrite Eq. (1) as

$$
E=\dot{\varphi}_{n}^{2}+\dot{\alpha}_{n}^{2}-2 \cos \alpha_{n} \cos \varphi_{n}+\beta^{2}\left(\alpha_{n+1}-\alpha_{n}\right)^{2},
$$

where

$$
\beta^{2}=\frac{1}{8 \pi l D E_{J}}\left(\frac{\Phi_{0}}{2 \pi}\right)^{2} \equiv \frac{E_{\mathrm{EM}}}{E_{J}}
$$

characterizes the ratio $\left(E_{\mathrm{EM}} / E_{J}\right)$ of electromagnetic (EM) and Josephson energies.

In this paper, the electromagnetic field is treated as a classical wave. We also assume that its amplitude is small, i.e., $\alpha_{n} \ll 1$. This means that the magnetic flux per unit cell area $H_{y} D \times l$ is much smaller than $\Phi_{0}$. Under such assumptions, the Hamiltonian for a single qubit is

$$
\mathcal{H}=-\left(\frac{\partial}{\partial \varphi_{n}}\right)^{2}-\alpha_{n}^{2} 2\left(\cos \varphi_{n}+I / I_{c}\right) .
$$

We restrict the states of each qubit to either its ground state $|0\rangle$, with energy $E_{0}$, or its excited state $|1\rangle$, with energy $E_{1}$. This is justified due to the nonlinearity of the Josephson potential.

We are not concerned here with decoherence effects in the qubits. We concentrate exclusively on their interaction with the electromagnetic wave in the system. This idealization is justified as long as the coherence time exceeds the wave propagation time across a significant number of unit cell periods, which is the case in some of the most recent experiments. For example, in Ref. 19, the charge qubit coupled to a strip line had a dephasing time in excess of 200 ns (i.e., a dephasing rate of $5 \mathrm{MHz}$ ) and a photon loss rate from the cavity of $0.57 \mathrm{MHz}$. Compared to the qubit transition frequency of the order of the Josephson energy of $\sim 6 \mathrm{GHz}$, which provides the scale for the effects we consider here, these frequencies are very small. For this choice of parameters and for $D \sim l \sim 10 \mu \mathrm{m}$, the dimensionless velocity $\beta$ (i.e., the number of unit cells the EM wave propagates per time period $\left.2 \pi / \omega_{J}\right)$ can be estimated from Eq. (3) as $\beta$ $\sim 30$. Therefore, the effects of decoherence and decay can be neglected for the purposes of this paper. Moreover, the regime considered here also justifies the use of the continuum approximation [Eq. (8)].

In the absence of an EM field, the wave function of the system $\Psi_{n}$ is a sum,

$$
\Psi_{n}=C_{0}^{n 0}|0\rangle e^{i \varepsilon t / 2}+C_{1}^{n 0}|1\rangle e^{-i \varepsilon t / 2},
$$

where $C_{k}^{n 0}$ are constants and $\varepsilon$ is the dimensionless excitation energy,

$$
\varepsilon=\frac{E_{1}-E_{0}}{\hbar \omega_{J}} .
$$

In the presence of an EM field, the coefficients $C_{k}^{n}$ become time dependent, and, as follows from Eq. (4), these obey the relation $^{20}$

$$
i \frac{d C_{k}^{n}}{d t}=\alpha_{n}^{2} \sum_{m=1,2} V_{k m}^{n}(t) C_{m}^{n}(t),
$$

with the initial condition $C_{k}^{n}(t=0)=C_{k}^{n 0}$. Here,

$$
V_{k m}^{n}(t)=\left\langle k\left|\cos \varphi_{n}\right| m\right\rangle\left(E_{J} / \hbar \omega_{J}\right)
$$

are matrix elements of the field-qubit interaction, calculated in the Heisenberg basis

$$
\{|0\rangle \exp (i \varepsilon t / 2),|1\rangle \exp (-i \varepsilon t / 2)\} .
$$

Later, we will also use the time-independent matrix elements $V_{k m}^{n}=\left\langle k\left|\cos \varphi_{n}\right| m\right\rangle\left(E_{J} / \hbar \omega_{J}\right)$ in the basis $\{|0\rangle,|1\rangle\}$.

By varying the energy [Eq. (2)], we obtain the equation for the electromagnetic field in the linear approximation as

$\ddot{\alpha}_{n}-\beta^{2}\left(\alpha_{n+1}+\alpha_{n-1}-2 \alpha_{n}\right)+\alpha_{n}\left\langle\Psi_{n}\left|\cos \varphi_{n}\right| \Psi_{n}\right\rangle\left(E_{J} / \hbar \omega_{J}\right)=0$.

The set of Eqs. (5)-(7) should be supplied by appropriate initial and boundary conditions. By controlling the qubits in Eq. (6), we propose to change the transmission and reflection of EM waves described in Eq. (7).

We are interested in the case when the wavelength is large compared to the size of the unit cell. Therefore, the qubit line can be treated as a continuous 1D medium, with $n \cdot l$ replaced by $x$. The difference [Eq. (7)] for $\alpha_{n}(t)$ and $\Psi_{n}(t)$ is thus replaced by a differential equation for $\alpha(x, t)$ and $\Psi(x, t)$,

$$
\begin{gathered}
\ddot{\alpha}-\beta^{2} \frac{\partial^{2} \alpha}{\partial x^{2}}+V_{0} \alpha=0, \\
V_{0}=\langle\Psi(x)|\cos \varphi(x)| \Psi(x)\rangle\left(E_{J} / \hbar \omega_{J}\right) .
\end{gathered}
$$

Within the perturbation theory approach, we present the electromagnetic wave as a sum of the larger incident wave $\alpha_{0}$ and the smaller scattered wave $\alpha_{1}$. A quantum state of the system is described by the wave function

$$
\Psi(x, t)=C_{0}(x, t)|0\rangle e^{i \varepsilon t / 2}+C_{1}(x, t)|1\rangle e^{-i \varepsilon t / 2} .
$$

In the unperturbed state, the coefficients in this equation are $C_{i}=C_{i}^{0}(x)$. We present the coefficients $C_{i}(x, t)$ as a sum of the 
unperturbed solution $C_{i}^{0}(x)$ and the small perturbation $C_{i}^{1}(x, t), C_{i}(x, t)=C_{i}^{0}(x)+C_{i}^{1}(x, t)$, with $\left|C_{i}^{1}\right| \ll 1$. Using Eq. (6), we derive the following:

$$
\begin{aligned}
& i C_{0}^{1}=\int_{0}^{t} d t^{\prime} \alpha_{0}^{2}\left(V_{00} C_{0}^{0}+V_{01} C_{1}^{0} e^{-i \varepsilon t^{\prime}}\right), \\
& i C_{1}^{1}=\int_{0}^{t} d t^{\prime} \alpha_{0}^{2}\left(V_{11} C_{1}^{0}+V_{10}^{*} C_{0}^{0} e^{i \varepsilon t^{\prime}}\right),
\end{aligned}
$$

where $V_{i k}=\langle i|\cos \varphi| k\rangle$ are calculated using the unperturbed wave functions, $V^{*}$ is the complex conjugate of $V$, and $V_{10}^{*}$ $=V_{01}$.

For the unperturbed EM wave $\alpha_{0}$, we obtain the following from Eq. (8)

$$
\ddot{\alpha}_{0}-\beta^{2} \frac{\partial^{2} \alpha_{0}}{\partial x^{2}}+V_{0} \alpha_{0}=0
$$

Here, $\left(V_{0}\right)^{1 / 2}$ plays the role of the Josephson plasma frequency, which is now controlled by the quantum state and quantum dynamics of the qubits. For the matrix element $V_{0}$, we can derive the following expression:

$$
V_{0}=\left|C_{0}^{0}\right|^{2} V_{00}+\left|C_{1}^{0}\right|^{2} V_{11}+C_{0}^{0} C_{1}^{0 *} e^{i \varepsilon t} V_{10}+\text { H.c. }
$$

For simplicity, we assume that $\alpha_{0}$ is a standing wave $\alpha_{0}$ $=A \cos (\omega t) \cos [k(\omega) x]$.

\section{ELECTROMAGNETIC WAVE PROPAGATION THROUGH A UNIFORM QUBIT LINE}

\section{A. Qubits initially in the ground state $|0\rangle$}

If all the qubits are in the ground state $|0\rangle$, then, initially, $C_{0}^{0}=1$ and $C_{1}^{0}=0$. In this case, $V_{0}=V_{00}$, and the wave vector is

$$
k(\omega)=\frac{1}{\beta} \sqrt{\omega^{2}-V_{00}} .
$$

Thus, the wave can propagate if its frequency exceeds $\left(V_{00}\right)^{1 / 2}$, which can be interpreted as the "ground state" plasma frequency of the medium. From Eq. (10), we obtain the following:

$$
\begin{gathered}
\frac{C_{0}^{1}(x, t)}{V_{00}}=-\frac{i A^{2} \cos ^{2}(k x)}{2}\left\{t+\frac{\sin (2 \omega t)}{2 \omega}\right\}, \\
\frac{C_{1}^{1}(x, t)}{V_{01}}=-\frac{A^{2} \cos ^{2}(k x)}{2}\left\{\frac{e^{i \varepsilon t}-1}{\varepsilon}\right. \\
\left.+\frac{\varepsilon+e^{i \varepsilon t}[2 i \omega \sin (2 \omega t)-\varepsilon \cos (2 \omega t)]}{4 \omega^{2}-\varepsilon^{2}}\right\} .
\end{gathered}
$$

The initial disturbance of the wave function produces a disturbance $\alpha_{1}$ in the propagating wave. For this perturbation, using Eq. (8), we derive

$$
\ddot{\alpha}_{1}-\beta^{2} \frac{\partial^{2} \alpha_{1}}{\partial x^{2}}+V_{00} \alpha_{1}+\Delta V_{0} \alpha_{0}=0,
$$

where $\Delta V_{0}$ is the perturbation of the field-qubit coupling. By means of Eq. (14), we obtain

$$
\begin{aligned}
\Delta V_{0}(t)= & -\left|V_{01}\right|^{2} A^{2} \cos ^{2}(k x)\left\{\frac{1}{\varepsilon}\right. \\
& \left.-\frac{2\left(2 \omega^{2}-\varepsilon^{2}\right) \cos (\varepsilon t)+\varepsilon^{2} \cos (2 \omega t)}{\varepsilon\left(4 \omega^{2}-\varepsilon^{2}\right)}\right\} .
\end{aligned}
$$

We see that the electromagnetic wave is in resonance with the qubit line if its frequency is one-half of the interlevel distance, $\omega=\varepsilon / 2$. This is due to the term that is proportional to $\alpha^{2}$ in the Hamiltonian (4). Near the resonance, the condition $\left|C_{i}^{1}\right| \ll 1$ is no longer valid and the usual perturbation approach fails.

\section{B. Qubits initially in the excited state $|\mathbf{1}\rangle$}

If all qubits are initially in the excited state $|1\rangle$, the solution will be in complete analogy to the previous case. As can readily be seen, we should only exchange $0 \leftrightarrow 1$ and $\varepsilon \leftrightarrow-\varepsilon$ in Eqs. (13)-(16),

$$
\begin{gathered}
k(\omega)=\frac{1}{\beta} \sqrt{\omega^{2}-V_{11}}, \\
\frac{C_{1}^{1}(x, t)}{V_{11}}=-\frac{i A^{2} \cos ^{2}(k x)}{2}\left\{t+\frac{\sin (2 \omega t)}{2 \omega}\right\}, \\
\frac{C_{0}^{1}(x, t)}{V_{10}}=-\frac{A^{2} \cos ^{2}(k x)}{2}\left\{\frac{1-e^{-i \varepsilon t}}{\varepsilon}\right. \\
\left.+\frac{-\varepsilon+e^{-i \varepsilon t}[2 i \omega \sin (2 \omega t)+\varepsilon \cos (2 \omega t)]}{4 \omega^{2}-\varepsilon^{2}}\right\} .
\end{gathered}
$$

For the electromagnetic wave, we obtain

$$
\ddot{\alpha}_{1}-\beta^{2} \frac{\partial^{2} \alpha_{1}}{\partial x^{2}}+V_{11} \alpha_{1}+\Delta V_{1} \alpha_{0}=0 .
$$

In Eq. (19),

$$
\Delta V_{1}(t)=-\Delta V_{0}(t),
$$

where $\Delta V_{0}(t)$ is given by Eq. (16).

The excited qubit line is an active medium, and one should expect a resonance pumping of the electromagnetic wave as it propagates along this medium. However, $\Delta V_{1}$ $\rightarrow 0$ at $2 \omega \rightarrow \varepsilon$ (and $\Delta V_{0} \rightarrow 0$ at $2 \omega \rightarrow \varepsilon$ as well). This "paradox" only reflects the limitations of the first-order perturbation approximation, where $\left|C_{i}^{0}+C_{i}^{1}\right|^{2}=\left|C_{i}^{0}\right|^{2}$. In other words, to first order, the qubit energy does not change. To describe the pumping effect, we must take into account the higher order terms, which is beyond the scope of our current investigation.

In the case of $C_{0}=C_{1}=1 / 2$, all qubits rotate between the ground and excited states. The matrix element in Eq. (8) is now 


$$
V_{0}(t)=\frac{1}{4}\left[V_{00}+V_{11}+2 V_{01} \cos (\varepsilon t)\right]
$$

Let us now assume, for simplicity, that the frequency of the electromagnetic wave is high, i.e., $\omega \gg \varepsilon$. Then, its wave vector is a slowly oscillating function,

$$
k(\omega, t) \approx \sqrt{\omega^{2}-\frac{V_{00}+V_{11}+2\left|V_{01}\right| \cos (\varepsilon t)}{4 \beta^{2}}} .
$$

If the wave frequency $\omega$ is close to the threshold value,

$$
\omega_{c}=\sqrt{V_{00}+V_{11}} /(2 \beta),
$$

then the qubit line will alternate between transparent and reflecting states with frequency $\varepsilon$ as the wave vector $k(t)$ switches between real and imaginary values. In addition, the qubit line will produce electromagnetic waves with frequencies $\varepsilon$ and $\omega \pm \varepsilon$.

\section{ELECTROMAGNETIC WAVE AT RESONANCE}

Near the resonance, $2 \omega-\varepsilon \equiv \Delta \omega \ll \omega$, we can use a resonant perturbation approach ${ }^{20}$ instead of a first-order perturbation approximation. In doing so, we drop all the terms in Eq. (6) except the resonant ones. As a result, we have

$$
\begin{gathered}
i \dot{C}_{0}=-\Omega e^{i \Delta \omega t} C_{1}, \\
i \dot{C}_{1}=-\Omega^{*} e^{-i \Delta \omega t} C_{0},
\end{gathered}
$$

where

$$
\Omega(x)=A^{2} \cos ^{2}[k(\omega) x] V_{01} / 4 .
$$

The solution of this system is

$$
\begin{gathered}
C_{0}(t)=\frac{e^{i \Delta \omega t / 2}}{\Omega^{*}}\left[g_{1}\left(\Lambda-\frac{\Delta \omega}{2}\right) e^{i \Lambda t}-g_{2}\left(\Lambda+\frac{\Delta \omega}{2}\right) e^{-i \Lambda t}\right] \\
C_{1}(t)=e^{-i \Delta \omega t / 2}\left(g_{1} e^{i \Lambda t}+g_{2} e^{-i \Lambda t}\right),
\end{gathered}
$$

where

$$
\Lambda=\sqrt{\Omega^{2}+(\Delta \omega)^{2} / 4},
$$

and $g_{1}$ and $g_{2}$ are constants. The coefficients $C_{i}$ satisfy the normalization condition $\left|C_{0}\right|^{2}+\left|C_{1}\right|^{2}=1$. If at $t=0$ the system were in the ground state, we would obtain

$$
\begin{gathered}
C_{0}(t)=e^{i \Delta \omega t / 2}\left[\cos (\Lambda t)-\frac{i \Delta \omega}{2 \Lambda} \sin (\Lambda t)\right], \\
C_{1}(t)=-\frac{i|\Omega|}{\Lambda} e^{-i \Delta \omega t / 2} \sin (\Lambda t) .
\end{gathered}
$$

At resonance $\Delta \omega=0$,

$$
\Psi=\exp (i \varepsilon t / 2) \cos (|\Omega| t)|0\rangle-i \exp (-i \varepsilon t / 2) \sin (|\Omega| t)|1\rangle,
$$

and each qubit periodically oscillates between its ground and excited states. The frequency of these transitions varies with the qubit position since $\Omega=\Omega(x)$. It can be considered as a spatially dependent quantum beat frequency. If the system were initially in its excited state, then we would have

$$
\begin{gathered}
C_{0}(t)=-\frac{i|\Omega|}{\Lambda} e^{i \Delta \omega t / 2} \sin (\Lambda t), \\
C_{1}(t)=e^{-i \Delta \omega t / 2}\left[\cos (\Lambda t)-\frac{i \Delta \omega}{2 \Lambda} \sin (\Lambda t)\right],
\end{gathered}
$$

and at $\Delta \omega=0$,

$\Psi=-i \exp (i \varepsilon t / 2) \sin (|\Omega| t)|0\rangle+\exp (-i \varepsilon t / 2) \cos (|\Omega| t)|1\rangle$.

Using these expressions for the wave functions, we find the matrix element $V_{0}$ in Eq. (12). Assuming for simplicity that $V_{i k}$ are real and $\Delta \omega^{2} \ll|\Omega|^{2}$, we obtain

$$
\begin{aligned}
V_{0}(t)= & \frac{V_{00}+V_{11}}{2} \pm \frac{V_{00}-V_{11}}{2} \cos (2|\Omega| t) \\
& -V_{01} \sin (2|\Omega| t) \sin (\varepsilon t+\Delta \omega),
\end{aligned}
$$

where the signs + and - correspond to the initial ground and excited states for qubits, respectively. If the transitions between the ground and excited states are suppressed $\left|V_{01}\right|$ $\ll\left|V_{00}-V_{11}\right|$, then, in resonance, the electromagnetic wave $\alpha_{0}$ will have a time-dependent wave vector,

$$
k(\omega, t)=\sqrt{\omega^{2}-V_{0}(t)},
$$

where $V_{0}(t)$ varies between $V_{00}$ and $V_{11}$. These results are valid if the wave-qubit interaction does not distort too much, $\left|\alpha_{1}^{2}\right| \ll\left|\alpha_{0}^{2}\right|$. In any case, the condition

$$
|\Omega| \sim A^{2}\left|V_{01}\right| \ll \omega \sim \varepsilon
$$

must be fulfilled.

\section{QUANTUM METAMATERIAL PHOTONIC CRYSTAL}

In analogy with photonic crystals, ${ }^{21}$ the interaction of the electromagnetic wave with qubits can produce a frequency gap in the spectrum of the propagating wave if the qubit states are periodically modulated in space. For example, supposing that the qubits are in the $|\gamma\rangle$ or $|\delta\rangle$ state with a spatial period $2 L$, the wave obeys

$$
\ddot{\alpha}-\beta^{2} \alpha_{x x}+V_{\gamma \gamma} \alpha=0
$$

or

$$
\ddot{\alpha}-\beta^{2} \alpha_{x x}+V_{\delta \delta} \alpha=0 .
$$

The $|\gamma\rangle$ and $|\delta\rangle$ can be either stationary states (eigenstates of the qubit Hamiltonian) or their superpositions. In the latter case, the photonic crystal discussion makes sense only if the quantum beat frequency is small compared to the frequency of the propagating wave, that is, $\varepsilon^{2} \ll\left|V_{00}\right|$ and $\left|V_{11}\right|$.

Following the usual band-theory approach for electrons in a crystal lattice, we seek the solution of Eqs. (28) and (29) in the form of a Bloch wave $\alpha(t, x)=u(x, k) \exp (i k x-i \omega t)$, where $u(x, k)$ is a periodic function of $x$ with the period $2 L$, and the dimensionless wave vector $k$ is in the first Brillouin zone, $-\pi / L<k<\pi / L$. Consider the $j$ th elementary cell of 


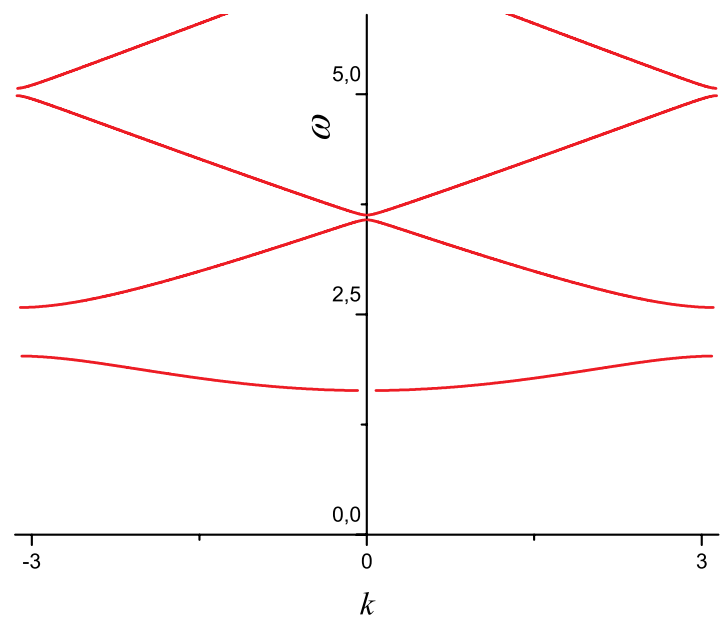

FIG. 2. (Color online) Photonic crystal spectrum obtained for a qubit transmission line. The $\omega(k)$ was calculated for a periodic array of qubit states (ground and excited). The ratio $V_{00} / V_{11}=5$.

our periodic structure: All the qubits are in state $|\gamma\rangle$ for $x_{j}$ $<x<x_{j}+L$ and in state $|\delta\rangle$ for $x_{j}+L<x<x_{j}+2 L$. In both regions, the solution $\alpha(t, x)$ of Eqs. (28) and (29) is a sum of exponential terms multiplied by constants $C_{j}$. By using the continuity of $\alpha$ and $\partial \alpha / \partial x$ at the boundaries of different regions and the periodicity of the Bloch functions $u(x, k)$, we obtain a set of homogeneous linear equations for $C_{j}$. The nontrivial solution of these equations exists only if the determinant of the set of equations is zero. Then, after straightforward algebra, we obtain the dispersion equation for the frequency $\omega(k)$ in the form

$$
\cos \left(\kappa_{\gamma} L\right) \cos \left(\kappa_{\delta} L\right)-\frac{\kappa_{\gamma}^{2}+\kappa_{\delta}^{2}}{2 \kappa_{\gamma} \kappa_{\delta}} \sin \left(\kappa_{\gamma} L\right) \sin \left(\kappa_{\delta} L\right)=\cos (2 k L),
$$

where

$$
\kappa_{\gamma}^{2}=\frac{\omega^{2}-V_{\gamma \gamma}}{\beta^{2}}, \quad \kappa_{\delta}^{2}=\frac{\omega^{2}-V_{\delta \delta}}{\beta^{2}} .
$$

This equation predicts the spectrum $\omega(k)$ with gaps if the difference between $\kappa_{\gamma}$ and $\kappa_{\delta}$ is large enough; that is, $\mid \kappa_{\gamma}^{2}$ $-\kappa_{\delta}^{2} \geqslant 1$ or

$$
\left|V_{\gamma \gamma}-V_{\delta \delta}\right| \gtrsim \beta^{2} .
$$

Thus, in order to form a photonic crystal in the qubit line, the Josephson energy $E_{J}$ must be large compared to the magnetic energy or, according to Eq. (3), must be

$$
E_{J} \gg \frac{1}{8 \pi}\left(\frac{\Phi_{0}}{\pi D l}\right)^{2} \text {. }
$$

The characteristic dependence of $\omega(k)$ is shown in Fig. 2. Note that the gap value for the first zone is of the order of unity if condition (32) is valid.

The gap depends on the quantum state of the qubits, making this a quantum photonic crystal. Changing the microscopic quantum state of the qubits changes the macroscopic electromagnetic response of the system.

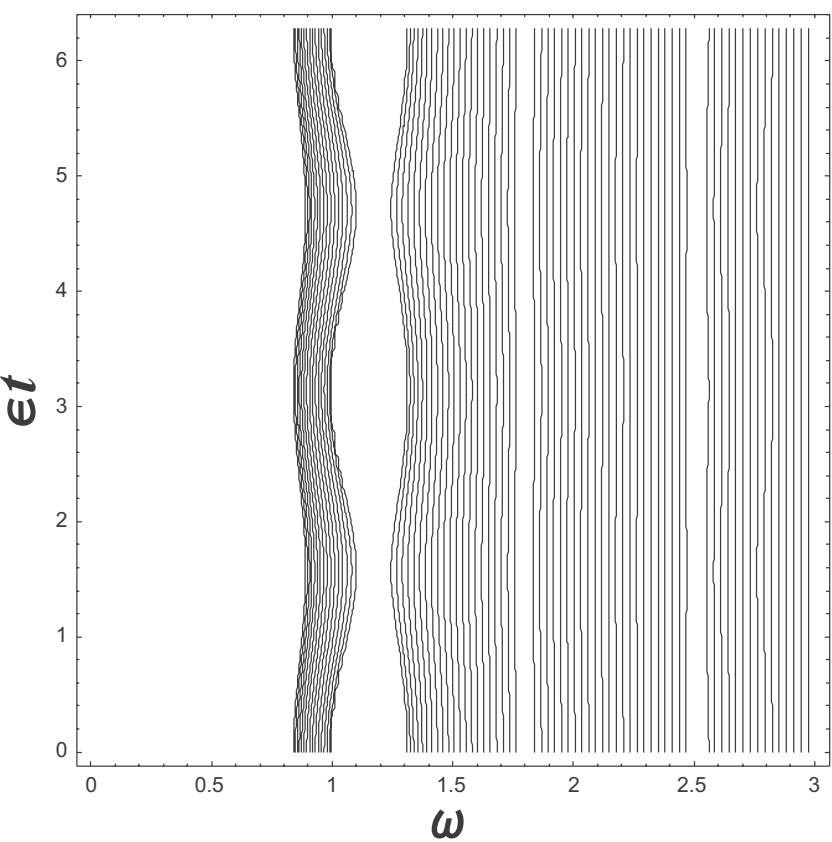

FIG. 3. Breathing photonic crystal: contour curves of the wave vector $k$ as a function of $\omega$ and $\varepsilon t$ in the situation described by Eq. (34). The parameters used here are $V_{00}=V_{01}=1, V_{11}=2$ (units of the qubit Josephson energy $\left.E_{J}\right), \beta=0.5$, and $L=2$. The time-dependent gaps in the spectrum are clearly seen.

A more interesting situation arises if one or both of the qubit states are not the eigenstate $|0\rangle$ or $|1\rangle$. In this case, the system would exhibit quantum beats between the two. Let, e.g., $|\gamma\rangle=|0\rangle$ and

$$
|\delta\rangle=\left\{|0\rangle e^{i \varepsilon t}+|1\rangle e^{-i \varepsilon t}\right\} / 2,
$$

then $V_{\gamma \gamma}=V_{00}$ and

$$
V_{\delta \delta}(t)=\left[V_{00}+V_{11}+2 V_{01} \cos (\varepsilon t)\right] / 4 .
$$

In this case, the photonic crystal arises if any of the matrix elements is of the order of unity. The frequency gap is modulated by the value of $V_{01} / 2$ with the period $\Delta t=2 \pi / \varepsilon$. If $V_{01} \sim 1$, then the modulation is significant. If

$$
|\gamma\rangle=\left\{|0\rangle e^{i \varepsilon t}-|1\rangle e^{-i \varepsilon t}\right\} / 2
$$

and

$$
|\delta\rangle=\left\{|0\rangle e^{i \varepsilon t}+|1\rangle e^{-i \varepsilon t}\right\} / 2,
$$

then

$$
\begin{aligned}
& V_{\gamma \gamma}(t)=\left[V_{00}+V_{11}-2 V_{01} \cos (\varepsilon t)\right] / 4, \\
& V_{\delta \delta}(t)=\left[V_{00}+V_{11}+2 V_{01} \cos (\varepsilon t)\right] / 4 .
\end{aligned}
$$

In this case, the photonic crystal appears if $V_{01} \sim 1$. The gap is strongly modulated in time from zero, at $t=(2 n$ +1) $\pi / 2 \varepsilon$, to its maximum value when $t=n \pi / \varepsilon$ (here, $n$ is an integer). Thus, we obtain an interesting uniformly breathing photonic crystal, as shown in Fig. 3. 


\section{QUANTUM ARCHIMEDEAN SCREW}

Let an external source produce a slow "control wave" propagating along the qubit line. That is, let the coefficients $C_{i}$ in the wave function be

$$
C_{0}(x, t)=\sin \left(\omega_{0} t-k_{0} x\right), \quad C_{1}(x, t)=\cos \left(\omega_{0} t-k_{0} x\right),
$$

where $\omega_{0}$ and $k_{0}$ are the frequency and the wave vector of the control wave, respectively. Both these values are small compared with the electromagnetic wave frequency and wave vector. Such a wave can be produced, e.g., by applying an rf signal to the qubits with a position-dependent phase and inducing Rabi oscillations between their ground and excited states. In order for us to neglect the decoherence effects in the lowest approximation, the control signal frequency must still be much higher than the decay and dephasing rates, i.e., realistically in excess of several megahertz [see the discussion after Eq. (4)].

The matrix element $V_{0}$ in the wave equations then takes the form

$$
\begin{aligned}
V_{0}(x, t)= & \frac{V_{00}+V_{11}}{2}-\frac{V_{00}-V_{11}}{2} \cos \left[2\left(\omega_{0} t-k_{0} x\right)\right] \\
& +V_{01} \sin \left[2\left(\omega_{0} t-k_{0} x\right)\right] \cos \varepsilon t,
\end{aligned}
$$

where we now assume that $V_{01}$ is real. To simplify the problem, we now assume that $\left|V_{01}\right| \ll\left|V_{00} \pm V_{11}\right|$, and the last term, which describes the qubit relaxation, can be neglected. Thus, $V_{0}=V_{0}(\xi)$, where $\xi=\omega_{0} t-k_{0} x$.

The function $V_{0}(\xi)$ varies slowly and we can seek the solution of Eq. (8) in the form

$$
\alpha(t, \xi)=A(\xi) \sin \left[\omega_{0} t-\eta(\xi)\right]
$$

where the wave amplitude $A(\xi)$ varies slowly and we can neglect terms with $A^{\prime \prime}(\xi)$ (here, $f^{\prime} \equiv d f / d \xi$ ). By substituting Eq. (37) into Eq. (8) and separating the terms with $\sin \left[\omega_{0} t\right.$ $-\eta(\xi)]$ and $\cos \left[\omega_{0} t-\eta(\xi)\right]$, we derive two equations for the wave amplitude $A$ and the phase $\eta$ in the following form:

$$
\begin{gathered}
\frac{2 A^{\prime}}{A}=-\frac{\left(\beta^{2} k_{0}^{2}-\omega_{0}^{2}\right) \eta^{\prime \prime}}{\left(\beta^{2} k_{0}^{2}-\omega_{0}^{2}\right) \eta^{\prime}+\omega \omega_{0}}, \\
\left(\eta^{\prime}\right)^{2}+2 \eta^{\prime} \frac{\omega \omega_{0}}{\beta^{2} k_{0}^{2}-\omega_{0}^{2}}+\frac{V_{0}-\omega^{2}}{\beta^{2} k_{0}^{2}-\omega_{0}^{2}}=0 .
\end{gathered}
$$

These equations are valid if

$$
\begin{gathered}
\left|\frac{A^{\prime \prime}}{A}\right| \ll\left(\eta^{\prime}\right)^{2}, \\
\left|\frac{A^{\prime \prime}}{A}\right| \ll \frac{\left(\omega-\omega_{0} \eta^{\prime}\right)^{2}}{\left(\omega_{0} \eta^{\prime}\right)^{2}} .
\end{gathered}
$$

By integrating the first of these equations, we get

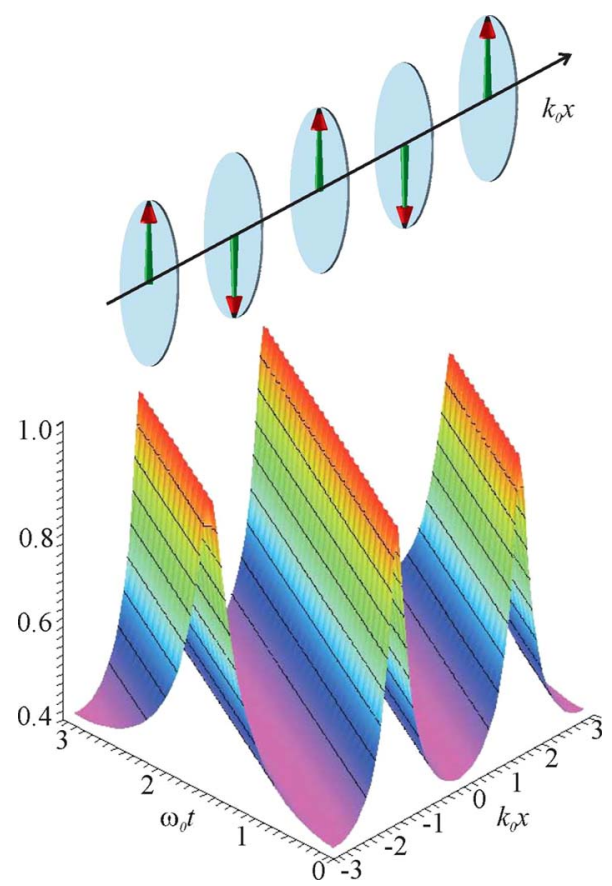

FIG. 4. (Color online) Quantum Archimedean screw: (top) schematic diagram of how the wave amplitude $A(x, t) / A_{0}$ varies with position at a given moment in time and (bottom) the dependence of the wave amplitude on time and position. $V_{00}+V_{11}=2, V_{00}-V_{11}$ $=1$ (units of the qubit Josephson energy $E_{J}$ ), $\beta=0.15, k_{0}=0.1, \omega_{0}$ $=10^{-3}$, and $\omega=1.23$.

$$
2 \ln A=-\ln \left(\eta^{\prime}+\frac{\omega \omega_{0}}{\beta^{2} k_{0}^{2}-\omega_{0}^{2}}\right)+\text { const. }
$$

The second equation is a quadratic equation for $\eta^{\prime}$. By choosing its positive root and substituting it into Eq. (40), we get

$$
\frac{A}{A_{0}}=\left[\frac{\left(\beta^{2} k_{0}^{2}-\omega_{0}^{2}\right)^{2}}{\beta^{2} k_{0}^{2} \omega^{2}-\left(\beta^{2} k_{0}^{2}-\omega_{0}^{2}\right) V_{0}}\right]^{1 / 4},
$$

where $A_{0}$ is a constant defined by the boundary conditions. The dependence $A(x, t) / A_{0}$ is shown in Fig. 4 for parameters chosen within the validity range [Eq. (39)]. It is easy to see that the wave amplitude $A(t)$ achieves its maximum if the denominator in Eq. (41) becomes small at some moments in time. The value $\beta^{2} k_{0}^{2} \omega^{2}-\left(\beta^{2} k_{0}^{2}-\omega_{0}^{2}\right) V_{0}$ should be positive. Assuming that $V_{00}>V_{11}$, we can write the last condition in the form

$$
\omega^{2}>\omega_{c}^{2}=V_{11}\left(1-\frac{\omega_{0}^{2}}{\beta^{2} k_{0}^{2}}\right) .
$$

The variation of $A(x, t) / A_{0}$ is maximum if $\omega$ is close to $\omega_{c}$; however, the conditions in Eq. (39) should be fulfilled.

Returning to Fig. 4, we see that the maxima of the fastoscillating electromagnetic field are transferred through the system at a pace set by the much slower control frequency $\omega_{0}$, which reminds us of an Archimedean screw or a meat grinder. 


\section{CONCLUSIONS}

Here, we show that for a classical electromagnetic wave, a line of qubits inside a superconducting cavity plays the role of a 1D transmission line with interesting characteristics and physics. In particular, the quantum superposition of qubit states produces a breathing state with transparency changing with the quantum beat frequency of a single qubit. More interestingly, a periodic arrangement of qubit states yields a quantum photonic crystal, which can also be put into a breathing mode. A time-domain control of the qubits allows us to realize an Archimedean screw state, where the incident electromagnetic wave is periodically modulated, and the regions of its maximum amplitude are carried along the qubit line with a desired speed. The investigation of the action of this system as an active medium requires a special treatment beyond the lowest-order perturbation theory and will be the subject of future research.

\section{ACKNOWLEDGMENTS}

This work was supported in part by the National Security Agency (NSA), Laboratory Physical Science (LPS), U.S. Army Research Office (USARO), National Science Foundation (NSF) Grant No. EIA-0130380, and JSPS-RFBR Grant No. 06-02-91200. A.Z. is grateful to J. Young for fruitful discussions and to the Natural Sciences and Engineering Research Council of Canada (NSERC) Discovery Grant Program for partial support. S.S. acknowledges support from the Ministry of Science, Culture and Sports of Japan via a Grantin-Aid for Young Scientists (No. 1874002), the EPSRC-GB via ARF. No. EP/D072581/1, and the ESF network program "Arrays of Quantum Dots and Josephson Junctions." We are grateful to A. Satanin for a careful reading of the manuscript and helpful remarks.
${ }^{1}$ J. Q. You and F. Nori, Phys. Today 58 (11), 42 (2005).

${ }^{2}$ G. Wendin and V. S. Shumeiko, in Handbook of Theoretical and Computational Nanotechnology, edited by M. Rieth and W. Schommers (American Scientific, New York, 2006), Vol. 3.

${ }^{3}$ A. Blais, A. Maassen van den Brink, and A. M. Zagoskin, Phys. Rev. Lett. 90, 127901 (2003).

${ }^{4}$ J. Q. You and F. Nori, Phys. Rev. B 68, 064509 (2003).

${ }^{5}$ J. Q. You, J. S. Tsai, and F. Nori, Phys. Rev. B 68, 024510 (2003).

${ }^{6}$ A. Wallraff, D. I. Schuster, A. Blais, L. Frunzio, R.-S. Huang, J. Majer, S. Kumar, S. M. Girvin, and R. J. Schoelkopf, Nature (London) 431, 162 (2004).

${ }^{7}$ A. M. Zagoskin, M. Grajcar, and A. N. Omelyanchouk, Phys. Rev. A 70, 060301(R) (2004).

${ }^{8}$ J. Q. You, Yu-xi Liu, C. P. Sun, and F. Nori, Phys. Rev. B 75, 104516 (2007).

${ }^{9}$ J. Hauss, A. Fedorov, C. Hutter, A. Shnirman, and G. Schön, Phys. Rev. Lett. 100, 037003 (2008).

${ }^{10}$ E. Il'ichev, N. Oukhanski, A. Izmalkov, Th. Wagner, M. Grajcar, H.-G. Meyer, A. Y. Smirnov, A. Maassen van den Brink, M. H. S. Amin, and A. M. Zagoskin, Phys. Rev. Lett. 91, 097906 (2003).

${ }^{11}$ S. Savel'ev, V. A. Yampol'skii, and F. Nori, Phys. Rev. Lett. 95, 187002 (2005); Physica C 445-448, 183 (2006); S. Savel'ev, A. L. Rakhmanov, V. A. Yampol'skii, and F. Nori, Nat. Phys. 2, 521 (2006); S. Savel'ev, A. L. Rakhmanov, and F. Nori, Phys. Rev. Lett. 94, 157004 (2005); Physica C 445-448, 180 (2006); S. Savel'ev, V. A. Yampol'skii, A. L. Rakhmanov, and F. Nori, Phys. Rev. B 72, 144515 (2005); Physica C 437-438, 281 (2006); 445-448, 175 (2006); V. A. Yampol'skii, S. Savel'ev, O.
V. Usatenko, S. S. Mel'nik, F. V. Kusmartsev, A. A. Krokhin, and F. Nori, Phys. Rev. B 75, 014527 (2007); S. Savel'ev, A. L. Rakhmanov, and F. Nori, Phys. Rev. Lett. 98, 077002 (2007); 98, 269901(E) (2007); S. Savel'ev, V. A. Yampol'skii, A. L. Rakhmanov, and F. Nori, Phys. Rev. B 75, 184503 (2007); S. Savel'ev, A. L. Rakhmanov, X. Hu, A. Kasumov, and F. Nori, ibid. 75, 165417 (2007).

${ }^{12}$ H. Salehi, R. R. Mansour, and A. H. Majedi, IET Proc. Microwaves, Antennas Propag. 1, 69 (2007).

${ }^{13}$ H. Salehi, A. H. Majedi, and R. R. Mansour, IEEE Trans. Appl. Supercond. 15, 996 (2005).

${ }^{14}$ M. C. Ricci, H. Xu, R. Prozorov, A. P. Zhuravel, A. V. Ustinov, and S. M. Anlage, IEEE Trans. Appl. Supercond. 17, 918 (2007).

${ }^{15}$ N. Lazarides and G. P. Tsironis, Appl. Phys. Lett. 90, 163501 (2007).

${ }^{16}$ Y. Wang and M. J. Lancaster, IEEE Trans. Appl. Supercond. 16, 1893 (2006).

${ }^{17}$ C. G. Du, H. Y. Chen, and S. Q. Li, Phys. Rev. B 74, 113105 (2006).

${ }^{18}$ M. Ricci, N. Orloff, and S. M. Anlage, Appl. Phys. Lett. 87, 034102 (2005)

${ }^{19}$ J. Gambetta, A. Blais, D. I. Schuster, A. Wallraff, L. Frunzio, J. Majer, M. H. Devoret, S. M. Girvin, and R. J. Schoelkopf, Phys. Rev. A 74, 042318 (2006).

${ }^{20}$ L. Landau and E. Lifshitz, Quantum Mechanics (ButterworthHeinemann, Oxford, 1995).

${ }^{21}$ B. E. A. Saleh and M. C. Teich, Fundamentals of Photonics (Wiley, New York, 2007). 